\title{
The Role of the N-Terminal Leucine Residue in Snake Venom Cardiotoxin II (Naja naja atra)
}

\author{
Chi-Yue Wu,*,1 Wan-Chen Chen,* Chewn-Lang Ho,* Shui-Tein Chen,* and Kung-Tsung Wang*, \\ *Institute of Biol ogical Chemistry, Academia Sinica, 128, Yan-Chiu-Yuan Road, Sec II, Nankang, Taipei, \\ Taiwan 11529, Republic of China; and †Department of Chemistry, National Taiwan University, 1, \\ Roosevelt Road, Sec IV, Taipe, Taiwan 11529, Republic of China
}

The $\mathbf{N}$-terminal leucine residue of snake venom cardiotoxin II (CTX II) (Naja naja atra) was systematically replaced with D-leucine (CTXII-L1-D-L), glycine (CTXII-L IG) or deleted [CTXII-(2-60)] to study the role of leucine residue in CTX II molecule. CTX II, CTXL 1$D-L, C T X L 1 G$ and CTX(2-60) were produced by chemical synthesis method and purified by high performance liquid chromatography. Owing to folding problem in CTXII-(2-60), only CTX II, CTXII-L1-D-L and CTXII-L 1G were produced in a pure form and characterized by amino acid analysis, mass spectrometry and peptide mapping. In thestructural aspect, changing the Leu-1 by D-Leu or Gly causes a drastic alteration in the whole CTX II structure as detected by circular dichroism, 1-anilino-naphthalene-8-sulfonate (ANS) fluorescence assay. In the functional aspect, both CTXII-L 1-D-L and CTXII-L1G are still retained substantial biological activity of CTX II. Therefore, the results indicate that both the chirality and the side-chain of the $\mathrm{N}$-terminal leucine residue of CTX II are important elements in maintaining the whole CTX II structure. In addition, this study is the first report in elucidating the reason why the first $\mathrm{N}$-terminal residue of most CTXs (90.3\%) is leucine residue. 1997 Academic Press

Snake venom cardiotoxins (CTXs) (also called cytotoxins), a group of basic proteins containing 60-63 amino acids and 4 disulfide bonds, are major lethal components of elapid snake venom $(1,2)$. In contrast to cobra neurotoxins, which interact with the postsynaptic acetylcholine receptor (3), CTXs show no defined cellular targets and have very diverse pharmacological

\footnotetext{
${ }^{1}$ To whom correspondence should be addressed: Chi-Y ue $\mathrm{Wu}, \mathrm{In}$ stitute of Biological Chemistry, Academia Sinica, 128, Yan-ChiuYuan Road, Sec II, Nankang, Taipei, Taiwan 11529. Fax: 886-27883473.

The abbreviations used are: ANS, 1-anilino-naphthalene-8-sulfonate; CTX, cardiotoxin; F moc, 9-fluorenylmethoxycarbonyl; HMP; 4-hydroxymethylphenoxymethylcopolystyrene-1\%-divinyl benzene; RP-HPLC, reverse-phase high performance liquid chromatography; TFA, trifluoroacetic acid.
}

effects, including lethal toxicity, hemolysis, cytolysis, muscle contractures, membrane depolarization, and activation of tissue phospholipase $C$ (4-7).

Among all CTXs, their $\mathrm{N}$-terminal residue is highly conserved and is either leucine (90.3\%) or isoleucine (7.3\%) except for CTX IV (Naja naja atra) $(5,8,9)$. Therefore, it is very interesting to explore the reason why the N-terminal residue of CTX is so highly conserved. In the present report, we choose CTX II (Naja naja atra) as a model molecule and systematically engineer its $\mathrm{N}$-terminal leucine residue by $\mathrm{D}$-leucine (CTXII-L 1-D-L), glycine (CTXII-LIG) or deleted [CTXII(2-60)] using chemical synthesis method as developed in our laboratory (10) to study the effects of $\mathrm{N}$-terminal substitutions on the structure and biological function of CTX II.

\section{MATERIALS AND METHODS}

Chemical synthesis. Samples were chemically synthesized using a F moc amino acid strategy with an Applied Biosystems 431A peptide synthesizer using the protocols and reagents provided by the manufacturers. The previously described procedures (10) were used to air-oxidize and purify each sample. The purity of each sample was determined by analytical RP-HPLC (Rainin, $\mathrm{C}_{18}, 5$ $\mu \mathrm{m}, 4.6 \times 250 \mathrm{~mm}$ ).

Determination of amino acid composition and molecular mass. Amino acid composition and molecular mass of each sample were determined as previously described (10). The extinction coefficients of $1 \%$ toxin solutions in water at $280 \mathrm{~nm}$ were 6.64 for CTX II and 6.60 for each CTX II analog.

Peptide mapping. Sample $(0.2 \mathrm{mg})$ was digested by thermolysin (5 units) in Tris- $\mathrm{HCl}$ buffer $\left(0.05 \mathrm{M}, \mathrm{pH} 7.5\right.$ ) at $37^{\circ} \mathrm{C}$ for $24 \mathrm{hr}$. Digested sample was analyzed by analytical RP-HPLC (Rainin, $\mathrm{C}_{18}, 5$ $\mu \mathrm{m}, 4.6 \times 250 \mathrm{~mm})$.

Circular dichroism spectra. Circular dichroism spectra were recorded at $25^{\circ} \mathrm{C}$ in quartz cells (path length $1 \mathrm{~mm}$ ) using a J ASCO model J -720 circular dichroism spectrophotometer equipped with a thermoelectric temperature controller and constantly flushed with nitrogen. The protein concentration was $0.23 \mathrm{mg} / \mathrm{mL}$ in $1 \mathrm{mM}$ Tris$\mathrm{HCl}$ buffer ( $\mathrm{pH}$ 8.0). Five scans were averaged for each of CTX samples and for the solvent.

ANS fluorescence spectra. Fluorescence spectra of ANS binding of samples were measured between 420 and $600 \mathrm{~nm}$ using the excitation wavelength of $400 \mathrm{~nm}$ on a Hitachi fluorescence spectrophotome- 


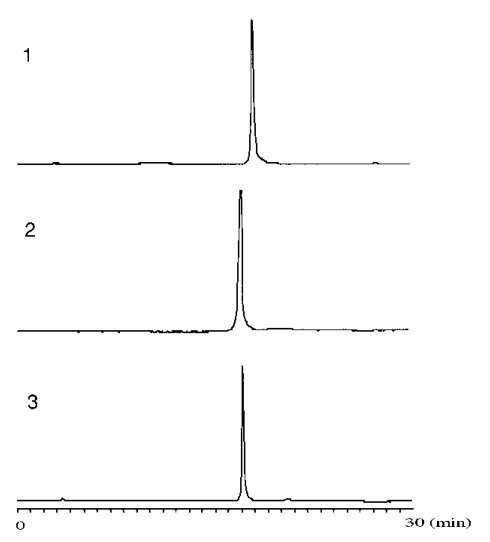

FIG. 1. HPLC profiles of purified CTX II and CTX analogs as analyzed by analytical RP-HPLC. (1) CTX II, (2) CTXII-L 1-D-L, and (3) CTXII-L 1G. Conditions: $\mathrm{C}_{18}$ column; $5 \%-95 \% \mathrm{CH}_{3} \mathrm{CN}$ containing $0.1 \%$ TFA, $30 \mathrm{~min} ; 1 \mathrm{ml} / \mathrm{min}$, UV $280 \mathrm{~nm}$.

ter, Model F-4010. The measurements were performed 10 min after mixing the sample $(10 \mu \mathrm{M})$ and ANS $(200 \mu \mathrm{M})$.

Biological activity assay. Lethal toxicity of CTX sample was measured by intravenous injection of sample into the tail veins of experimental mice (19-21g). Six mice were used at each dose, and the toxicity was expressed as $L_{50}(11)$. Six control mice were treated under the same conditions, but without CTX sample.

The ability of the CTX sample to stimulate muscle contractions was assayed using a chicken biventer cervices muscle preparation (12). The muscle preparation was maintained in $20 \mathrm{ml}$ Krebs solution and oxygenated with $95 \% \mathrm{O}_{2}$ and $5 \% \mathrm{CO}_{2}$ at $37^{\circ} \mathrm{C}$. The muscle was stimulated indirectly with supramaximal rectangular electric pulses of $0.5 \mathrm{~ms}$ duration at a frequency of $0.2 \mathrm{~Hz}$. The isometric contractions were recorded with a Grass FT 03 force displacement transducer attached to a Grass 7D polygraph. Toxins (1.5 $\mu \mathrm{M})$ were applied to the bathing medium to assess their ability to increase muscle contractions.

\section{RESULTS}

Synthesis of CTX II and its analoges. CTXII-(2-60) peptide-resins were first produced by coupling each amino acid residue from $\mathrm{C}$-terminal Asn-60 to $\mathrm{N}$-terminal Lys-2 on 4-hydroxymethyl-phenoxymethylcopolystyrene-1\%-divinylbenzene (HMP) resin. Consequently, CTX II, CTXII-LI-D-L and CTXII-LIG peptide-resins were produced by coupling the last $\mathrm{N}$ terminal residue, L-Leu, D-Leu, or Gly, respectively, onto CTXII-(2-60) peptide-resin. After 95\% trifluoroacetic acid (TFA) cleavage of each peptide-resin, each crude reduced CTX sample (purity about 30\%) was purified by semi-preparative $C_{18}$ RP-HPLC, and then the purified sample was directly air-oxidized in the phosphate buffer for 7 days (10). Each crude oxidized sample was purified by semi-preparative $C_{18}$ RP-HPLC and further purified by analytic $\mathrm{C}_{18}$ RP-HPLC. Unfortunately, owing to folding problem in CTXII-(2-60), only CTX II, CTXII-L 1-D-L and CTXII-LIG were obtained in a pure form (above 99\%), as shown in Fig. 1.

Characterization of CTX II and its analogs. Amino acid compositions of CTX samples were determined by amino acid analysis. The results show that values for each CTX sample can be consistent with the corresponding theoretical values of each amino acid residue except for cystine, methionine and tyrosine, as shown in Table 1. Molecular mass of each CTX sample was determined by electrospray mass spectroscopy. The results show that molecular masses of CTX II (6742.13), CTXII-L 1-D-L (6742.13) and CTXII-L IG (6686.02) are $6742.03,6742.69$ and 6687.00, respectively, as shown in Table 1. The molecular mass data are consistent with their corresponding theoretical value, respec-

TABLE 1

Amino Acid Compositions and Molecular Masses of CTX Samples

\begin{tabular}{|c|c|c|c|c|c|c|}
\hline \multirow[b]{2}{*}{ Amino acid } & \multicolumn{2}{|c|}{ CTX II } & \multicolumn{2}{|c|}{ CTXII-L 1-D-L } & \multicolumn{2}{|c|}{ CTXII-LIG } \\
\hline & Theoretical & Found & Theoretical & Found & Theoretical & Found \\
\hline Asp & 8 & 7.45 & 8 & 7.85 & 8 & 7.33 \\
\hline Thr & 3 & 2.50 & 3 & 2.37 & 3 & 2.07 \\
\hline Ser & 2 & 1.35 & 2 & 1.78 & 2 & 1.04 \\
\hline Pro & 4 & 3.62 & 4 & 3.71 & 4 & 3.73 \\
\hline Gly & 2 & 2.10 & 2 & 2.01 & 3 & 3.05 \\
\hline Ala & 2 & 2.00 & 2 & 2.00 & 2 & 2.00 \\
\hline Val & 7 & 6.10 & 7 & 6.40 & 7 & 6.25 \\
\hline Met & 2 & 0.20 & 2 & 0.17 & 2 & 0.12 \\
\hline Ile & 1 & 0.89 & 1 & 0.80 & 1 & 0.87 \\
\hline Leu & 6 & 5.14 & 6 & 5.30 & 5 & 4.50 \\
\hline Tyr & 3 & 1.56 & 3 & 1.95 & 3 & 1.86 \\
\hline Phe & 2 & 1.65 & 2 & 1.90 & 2 & 1.67 \\
\hline Lys & 8 & 7.22 & 8 & 7.20 & 8 & 7.03 \\
\hline Arg & 2 & 2.05 & 2 & 2.00 & 2 & 1.63 \\
\hline $1 / 2 \mathrm{CYSSCY}$ & 8 & 2.54 & 8 & 2.81 & 8 & 2.56 \\
\hline Molecular weight & 6742.13 & 6742.69 & 6742.13 & 6742.69 & 6686.02 & 6687.00 \\
\hline
\end{tabular}




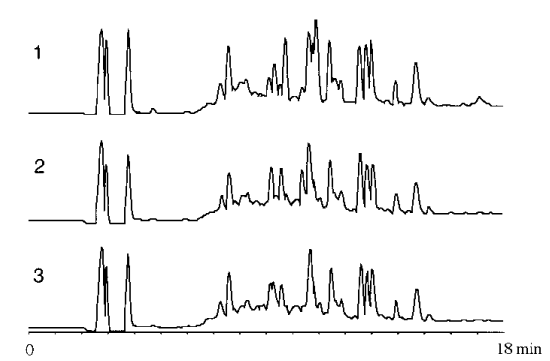

FIG. 2. HPLC profiles of digested (1) CTX II, (2) CTXII-L 1-D-L and (3) CTXL1G by protease thermolysin. Conditions: $\mathrm{C}_{18}$ column; $5 \%-95 \% \mathrm{CH}_{3} \mathrm{CN}$ containing $0.1 \%$ TFA, $30 \mathrm{~min}$; $1 \mathrm{ml} / \mathrm{min}$, UV $280 \mathrm{~nm}$.

tively. In addition, peptide mapping was used to compare the disulfide-linkage patterns of these CTX samples. The results show that the peak patterns in HPLC profiles of thermolysin-digested CTX II , CTXII-L 1-D-L and CTXII-LIG are similar to each other except for a few minor peaks which are resulted from the first $\mathrm{N}$ terminal residue differences among these CTX analogs, as shown in Fig. 2. This result suggests that the disulfide-linkage patterns of these CTX analogs are similar to each other.

Structural comparison of CTX II and its analogs. Circular dichroism and ANS fluorescence assays were used to compare the tertiary structures of CTX II, CTXII-LI-D-L and CTXII-LIG.

In the far ultraviolet region of circular dichroism spectrum, CTX II displays a negative trough at 211.0

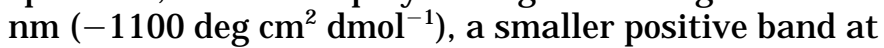
$224.1 \mathrm{~nm}\left(600 \mathrm{deg} \mathrm{cm}^{2} \mathrm{dmol}^{-1}\right)$, and a larger positive peak at $195.0 \mathrm{~nm}$ (400 deg $\mathrm{cm}^{2} \mathrm{dmol}^{-1}$ ), indicating predominant $\beta$-sheet organization, as shown in Fig. 3. The negative trough presented in CTX II is shifted from

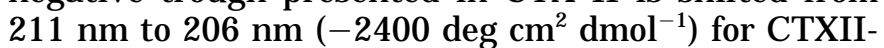
L 1-D-L and to $204 \mathrm{~nm}\left(-4400 \mathrm{deg} \mathrm{cm}^{2} \mathrm{dmol}^{-1}\right)$ for CTXII-L 1G. The two positive peaks presented in CTX

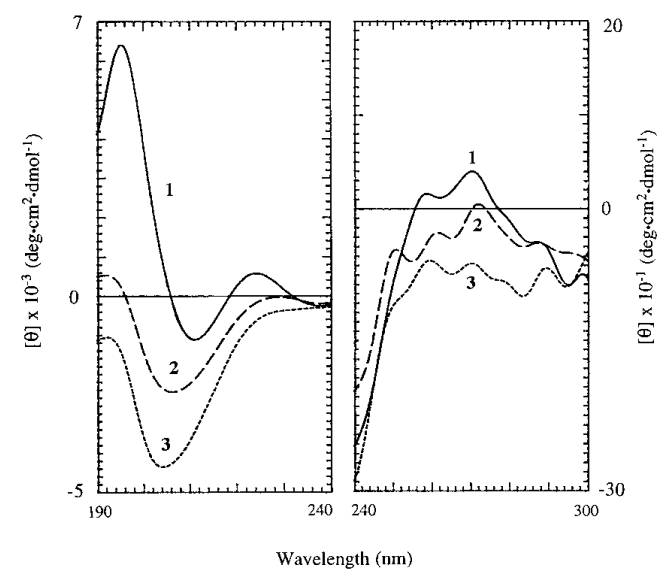

FIG. 3. Circular dichroism spectra of (1) CTX II, (2)CTXII-L 1-D$\mathrm{L}$ and (3) CTXL1G.

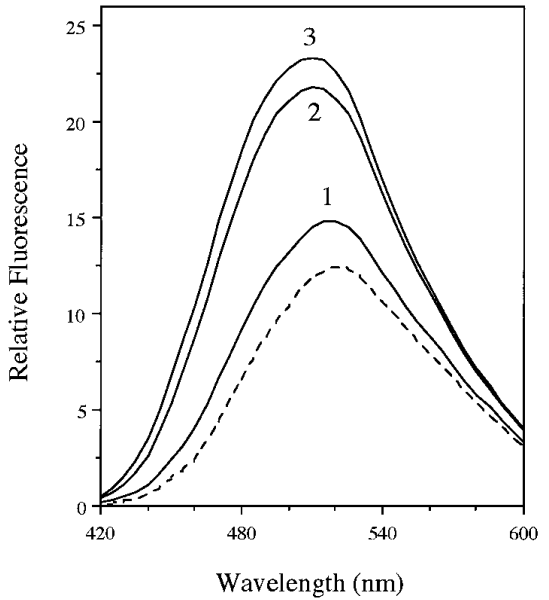

FIG. 4. Fluorescence spectra of ANS in the presence of various CTX samples. Curves: (1) CTX II, (2)CTXII-L 1-D-L and (3) CTXL1G. The dotted line shows the fluorescence of ANS in the absence of sample.

II are markedly decreased for CTXII-L 1-D-L and CTXII-L IG spectra. In the near ultraviol et region, CTX II displays a broad positive peak in the 256-277 nm wavelength range. This region is much decreased for CTXII-LIG and is completely reverse to a negative trough for CTXII-L 1-D-L.

The fluorescence emission of ANS is known to increase when it binds to hydrophobic regions of a protein (13). Therefore, ANS is a suitable hydrophobic probe for comparison of the accessibility of the hydrophobic core in CTX II and its analogs. Figure 4 shows the fluorescence spectra of ANS in the presence of various CTX samples. Only a limited increase in fluorescence is observed in the presence of CTX II, whereas a substantial fluorescence increase is observed in the presence of CTXII-L 1-D-L or CTXII-LIG. In addition, the wavelength of maximum emission is shifted from 520 $\mathrm{nm}$ in CTX II to $510 \mathrm{~nm}$ in both CTX II analogs. This result indicates that the ANS molecule has become enclosed in a more hydrophobic environment in CTXIIL 1-D-L or CTXII-L 1G than CTX II (14). Thus, the consequence of ANS fluorescence analysis suggests that there are more accessible hydrophobic regions of both CTXL1-D-L and CTXL1G for ANS than CTX II.

Biological activities of CTX II and its analogs. Lethal toxicity and muscle contracture assays were used to evaluate the biological activity of each CTX sample. Lethal toxicity (expressed as LD $_{50}$ ) of CTX II, CTXIIL1-D-L and CTXII-LIG are 2.47, 4.32 and $6.77 \mu \mathrm{g} / \mathrm{g}$, respectively, as shown in Table 2 . In muscle contracture assay, $1.5 \mu \mathrm{M}$ CTXII-L1-D-L and CTXII-L 1G stimulated muscle contraction with a force of 200 and $170 \mathrm{mg}$, respectively, approximately $26 \%$ and $22 \%$, respectively, of force elicited by $1.5 \mu$ M CTX II (780 mg). 
TABLE 2

Biological Activities of CTX II and Its Analogs

\begin{tabular}{lcc}
\hline \multicolumn{1}{c}{ Toxin } & $\begin{array}{c}\text { LD }_{50} \\
(\mu \mathrm{g} / \mathrm{g} \text { body wt) }\end{array}$ & $\begin{array}{c}\text { Muscle contracture } \\
(\mathrm{mg})\end{array}$ \\
\hline CTX II & $2.47(2.34-2.62)$ & $780 \pm 130(3)$ \\
CTXII-L 1-D-L & $4.32(4.11-4.53)^{*}$ & $200 \pm 10(3)^{*}$ \\
CTXII-LIG & $6.77(6.54-7.01)^{*}$ & $170 \pm 40(3)^{*}$ \\
\hline
\end{tabular}

\footnotetext{
a Values given are means \pm S.E.M. with the numbers of experiments in parentheses.

$* p<0.05$ as compared with the value of CTX II.
}

\section{DISCUSSION}

In our study, as comparison with the conformations of CTX II and CTXII-L 1-D-L, a significant structural difference is presented which is resulted from changing the chirality of Leu-1 in CTX II from the L- to the Dform. In the same way, as comparison of CTX II and CTXII-L 1G, a substantial structural difference is also presented which is resulted from the deletion of the side-chain of Leu-1 in CTX II. These results clearly indicate that both the chirality and the side-chain of the first $\mathrm{N}$-terminal Leu residue of CTX II are important el ements in the maintenance of CTX II conformation. Although the three-dimensional structure of CTX II (Naja naja atra) had been determined (15), only limited information about $\mathrm{N}$-terminal residue could be obtained which is not enough for interpreting our experimental results. Therefore, it is necessary to further determine the three-dimensional structures of both CTXII-L IG and CTXII-L 1-D-L to find out more data to elucidate why changing the first residue causes the drastic alteration in the conformation of CTX II molecule. This work is in progress in our laboratory.

Owing to drastic tertiary structure differences in CTX II and it analogs, the functional role of N-terminal leucine residue in CTX II can not been elucidated unequivocally. However, according to these functional data, we can infer that the native conformation and the Leu-1 of CTX II are probably not essential for its biological activity.

In conclusion, the structural and functional analyses suggest that the $\mathrm{N}$-terminal leucine residue plays an important structural element. In addition, this report also provides a possible reason to interpret why the first N-terminal residue of most CTXs (90.3\%) is leucine residue. On the other hand, the present report is the first example of engineering CTX mutants using chemical synthesis, and this method may provide a feasible and efficient route to study the structure/function relationship of other biologically active polypeptides consisting of approximately 60 residues.

\section{ACKNOWLEDGMENTS}

We are grateful to Prof. Wen-Chang Chang of the Institute of Biological Chemistry, Academia Sinica, and Dr. Ming-Fai Tam of the Institute of Molecular Biology, Academia Sinica, for performing the mass spectrometry; and Prof. Shyh-Horhg Chiou and Ms. Hui-Ming $\mathrm{Yu}$ of the Institute of Biological Chemistry, Academia Sinica, for assisting with the measurement of fluorescence spectrum and the peptide synthesis, respectively. Support for this research provided by Academia Sinica and the National Science Council, Taipei, Taiwan is gratefully acknowledged.

\section{REFERENCES}

1. Fryklund, L., and Eacker, D. (1975) Biochemistry 14, 28652871.

2. Bougis, P. E., Marchot, P., and Rochat, H. (1986) Biochemistry 25, 7235- 7243 .

3. Changeux, J . P. (1981) Haevey Lect. 75, 85- 254.

4. Lee, C. Y. (1972) Ann. Rev. Pharmacol. 12, 265- 284.

5. Dufton, M. J ., and Hider, R. C. (1991) in Snake Venom (Harvey, A. L., Ed.), pp. 259-302, Pergamon Press, New York.

6. Harvey, A. L. (1991) Handbook of Natural Toxins, 5th ed., pp. 85-106, Dekker, New York.

7. Fletcher, J . E., and J iang, M. S. (1993) Toxicon 31, 669-695.

8. Chiou, S. H., Chuang, M. H., Hung, C. C., Huang, H. C., Chen, S. T., Wang, K. T., and Ho, C. L. (1995) Biochem. Mol. Biol. Int. 35, 1103- 1112.

9. Chen, K. Y., Huang, W. N., J ean, J . H., and Wu, W. G. (1991) J . Biol. Chem. 266, 3252- 3259.

10. Wu, C. Y., Chen, S. T., Ho, C. L., and Wang, K. T. (1996) J . Chin. Chem. Soc. 43, 67-71.

11. Litchfield, J.T., and Wilcoxon, F. (1949) J . Pharmacol. Exp. Ther. 96, 99- 113.

12. Ginsborg, B. L., and Warriner, J . (1960) Br. J . Pharmacol. 15, 410-411.

13. Stryer, L. (1965) J . Mol. Biol. 13, 482- 495.

14. Turner, D. C., and Brand, L. (1968) Biochemistry 7, 3381- 3390.

15. Bhaskaran, R., Huang, C. C., Tsai, Y. C., J ayaraman, G., Chang, D. K., and Yu, C. (1994) J . Biol. Chem. 269, 23,500-23,508. 